\title{
Non-infectious non-anterior uveitis: Pharmacological management
}

\author{
Luis Rodriguez-Rodriguez* \\ Grupo de Patología Musculoesquelética, Hospital Clínico San Carlos, and Instituto de Investigación Sanitatia San Carlos (IdISSC), Madrid, Spain
}

\begin{abstract}
Non-infectious non-anterior uveitis (NINA-U) includes a heterogeneous group of sight-threatening conditions. NINA-U can be incapacitating and associated with a profound impact in the quality of life and well-being of the patients. To prevent this catastrophic course, a quick, complete, and maintained suppression of the inflammatory manifestations is required. To achieve this objective, we count with several drugs, including corticosteroids and immunomodulatory drugs (IMDs). Corticosteroids are the cornerstone of NINA-U treatment due to their rapid effect in suppressing inflammation. They can be administered using different routes (periocular, intravitreal, or systemic) depending on the characteristics and location of the disease. Local corticosteroids are able to achieve a high bioavailability within the eye, with minimal systemic dissemination. They are associated with a higher risk of local adverse events (mostly cataracts and elevation of the intraocular pressure). Systemic corticosteroids are usually administered orally, although under certain circumstances, they can be administered intravenously. When corticosteroids alone are not able to control the disease; or when they are associated with serious adverse events; or when the disease is severe, IMDs can be combined with systemic and/or local corticosteroids. Several are used in clinical practice (cyclosporine, methotrexate, azathioprine, mycophenolate mofetil, tacrolimus, cyclophosphamide, and biological agents), based on evidence on their efficacy/effectiveness and safety generated in randomized controlled trials and observational studies, respectively. These drugs are usually used in monotherapy, although several studies have shown that a varying percentage of patients will require changing the IMD or adding a second drug in combination to successfully control the disease.
\end{abstract}

Key words: Uveitis. Corticosteroids. Immunomodulatory drugs. Clinical management.

\section{Epidemiology and burden of the uveitis}

Uveitis comprises a heterogeneous group of rare diseases characterized by inflammation of the middle layer of the eye wall and adjacent tissues, such as the vitreous and the retina. These conditions can be classified according to the primary anatomical location of the inflammation (anterior, intermediate, posterior, or panuveitis) ${ }^{1}$, which will determine the prognosis and recommended therapy for the disease. In addition, uveitis can also be classified based on their etiology: infectious, the most frequent in the developing world, and non-infectious uveitis (NIU), comprising most of the causes in the EU and the US².
Regarding the etiology of NIU, in recent years, there have been substantial advances in the understanding of their pathogenic mechanisms, pointing to the interplay between a complex genetic background ${ }^{3}$ and environmental factors, triggering a deregulated innate and adaptive immune response ${ }^{4,5}$, able to overcome the ocular immune privilege.

Uveitis are potentially sight-threatening diseases. The World Health Organization estimated that, worldwide, uveitis represented up to $10 \%$ of causes of blindness (almost 4 million people) ${ }^{6}$. In the EU and US, and after diabetic retinopathy, uveitis represents the second major treatable cause of blindness in those 20-65 years of $\mathrm{age}^{7}$, accounting for up to $10 \%$ of cases of blind
Correspondence:

*Luis Rodriguez-Rodriguez

E-mail: Irrodriguez@salud.madrid.org
Available online: $21-10-2021$ J IMIDs. 2021;1(1):27-32

www.JournalofIMIDs.com
Accepted: 20-08-2021

DOI: 10.24875/JIMIDS.M21000003 
registrations ${ }^{8}$. In addition, more than $50 \%$ of the subjects affected with these conditions will develop complications related to the uveitis, and more than $30 \%$ will suffer visual impairment ${ }^{9}$. Furthermore, uveitis is associated with a negative impact in the subject's quality of life ${ }^{10}$. Because of the profound impact on patient's health and their higher prevalence in young- to middle-aged adults ${ }^{11}$, these conditions are associated with an important economic, social, and personal burden, resulting in work disability, reduced productivity, and work loss ${ }^{12}$.

Among NIU, those affecting more posterior segments of the eye (intermediate, posterior, or panuveitis) are associated with worse prognosis, greater risk of vision loss, ocular complications, and a greater impact in the subject's quality of life ${ }^{13}$, as they can affect structures that are essential for vision, such as the macula or the optic nerve. Because of this greater deleterious impact of non-infectious non-anterior uveitis (NINA-U), in this article, we will discuss the different medical treatments and strategies used to manage these conditions.

\section{Management of the NINA-U}

As pointed out, considering the potential negative impact of NINA-U on patients' health and well-being, their correct management is essential for preserving visual function and avoiding ocular and extraocular morbidity ${ }^{14}$. Patients are usually treated based on the presence and location of signs of inflammatory activity using a stepwise protocol to fully control the inflammation as soon as possible. Furthermore, it is fundamental to realize that most NINA-U are relapsing or chronic diseases that will require long-term treatment (sometimes for many years) to maintain a complete abrogation of the ocular inflammatory manifestations, since the presence of even low levels of residual inflammation or the occurrence of inflammatory relapses have been associated with worse visual prognosis ${ }^{15-17}$. At the same time, during the often prolonged treatment, local and systemic safety issues have to be considered, and, depending on the drugs prescribed, different measures and periodic assessments have to be carried out to prevent and early detect adverse events ${ }^{18}$.

\section{Corticosteroids}

These medications remain the gold standard agents in the management of NINA-U due to their immediate efficacy. Location and severity of the inflammation will influence their route of administration ${ }^{19-21}$. Although corticosteroids are able to carry out a rapid and complete suppression of the ocular inflammatory process, they are also associated with local and systemic adverse events, most of them related to a prolonged treatment with high dosages ${ }^{22}$.

To increase the bioavailability of corticosteroids at the inflamed tissue and decrease the systemic exposure (and therefore reducing the risk of systemic adverse events), alternative routes of drug delivery were developed, including periocular and intravitreal.

\section{Periocular}

Experimental studies have clearly demonstrated that posterior periocular corticosteroid injections produce high concentrations of active agent within the posterior segment of the eye, higher than systemic corticosteroids, particularly when the eye is inflamed ${ }^{23,24}$. This therapeutic option is useful in the management of posterior segment inflammation in NINA-U: several studies have shown an improvement in visual acuity and a reduction in inflammatory manifestations, including macular edema, when corticosteroids are administered locally ${ }^{25,26}$. They are particularly applicable to patients with active disease in one eye only; when there are specific contraindications to systemic corticosteroid therapy or combined with systemic therapy in case of persistent or refractory macular edema ${ }^{27}$. However, a major issue to long-term management of NINA-U with periocular corticosteroid injections is the limited duration of action (mean duration being 9 weeks ${ }^{23}$ ) that would require repeated injections, increasing the risk of local adverse events, typically elevation of intraocular pressure/glaucoma and cataract onset and progression ${ }^{26}$.

\section{Intravitreal}

This route of administration is able to achieve the highest intraocular corticosteroids bioavailability, as it bypasses the blood-retinal barrier and inoculates the drug directly in the eye. However, although this route has been successful in reducing macular edema and improving visual acuity, its duration of action is typically short term and associated with higher risk of corticoids related adverse events (even compared with periocular corticosteroids), such as ocular hypertension and cataracts. In addition, this route is associated with injection-related adverse events, including retinal detachment, vitreous and intraocular hemorrhage, and endophthalmitis ${ }^{28}$. 
To overcome the need for repeated and frequent injections to abrogate the inflammatory process, sustained release intravitreal corticosteroid implants have been developed, able to administer corticosteroids locally over an extended period overtime (months/ years). These implants are available in short-acting (dexamethasone implants) and long-acting forms (fluocinolone acetonide implants).

Dexamethasone implants are able to release corticosteroids for up to 6 months, and they have shown a reduction in inflammation and increase in visual acuity, when compared with sham injections in a randomized controlled trial ${ }^{29}$. It also showed improved effectivity when compared with periocular corticosteroids injections, measured as reduction of uveitis macular edema and increase of visual acuity ${ }^{30}$. However, no differences were observed when compared with intravitreal corticosteroids injections.

Regarding fluocinolone acetonide implants, they are able to release corticosteroids for up to 3 years with minimal systemic absorption. They have shown efficacy in reducing recurrences and improving visual acuity, when compared with the previous uveitis history of the patient $^{31}$. In addition, when compared with systemic corticosteroid therapy combined with immunomodulatory drugs (IMDs), they showed a similar control of inflammation ${ }^{32}$, although in the long term (up to 7 years follow-up), systemic therapy achieved better outcomes. The major caveat of this treatment are the local adverse events: almost all treated eyes required cataract surgery, three-fourths required intraocular pressure lowering medications, and almost half required glaucoma surgery ${ }^{31}$.

Recently, a fluocinolone acetonide insert has been developed, releasing corticosteroids at a lower dosage and for up to 3 years. It has shown efficacy in reducing the recurrence when compared with sham injections ${ }^{33}$, with lower risk of intraocular pressured hypertension and similar risk of cataract development and progression. No comparison with the fluocinolone acetonide implants has been performed so far.

\section{Systemic corticosteroids}

Systemic corticosteroids are used to treat severe inflammatory manifestations in the posterior parts of the eye. Initial recommended dosage is $1 \mathrm{mg} / \mathrm{kg} /$ day of oral prednisone or equivalent (up to $60 \mathrm{mg} /$ day). However, lower initial dosages could be used depending on the intensity and characteristics of the inflammatory manifestations ${ }^{34}$. After 2-4 weeks, the initial dosage is progressively tapered with the objective of maintaining a complete control of the inflammatory process at a prednisone dose of $\leq 7.5 \mathrm{mg} /$ day, a dosage that is associated with low risk of systemic and local adverse events ${ }^{35}$.

In case of vision-threatening diseases, with severe ocular inflammation, high dosages of intravenous corticosteroids can be administered in pulses (the traditional dose suggested is $1000 \mathrm{mg}$ of methylprednisolone per day for 3 consecutive days ${ }^{36}$ ). Several observational studies have shown that this strategy results in substantial improvement for most subjects treated and it was associated with low risk of ocular and systemic adverse events ${ }^{37,38}$. However, it is important to point out that in a comparative retrospective study in subjects with acute Vogt-Koyanagi-Harada disease, the route of administration (oral vs. intravenous) was not significantly associated with visual outcomes or the development of sight-threatening ocular complications ${ }^{39}$.

\section{IMDs}

In addition to corticosteroids, IMDs may be required to control the inflammatory manifestations of the NINA-U or to prevent the onset of ocular structural complications. The most frequent causes of IMD indication are related to the arise of issues with the local and/or systemic corticosteroid treatment: when they are not able to control the disease, when these medication are associated with intolerable adverse events (local or systemic), or when high dosages of systemic corticosteroids are needed to control inflammation (> 7.5/10 mg/day of prednisone or equivalent $)^{14}$. Furthermore, the presence of severe disease is also considered an indication for IMDs, such as bilateral uveitis; NINA-U with posterior, optic nerve or macular involvement; or a disease that severely impairs daily activities $^{14,40}$.

Several IMDs are currently prescribed as part of the standard of care of NINA-U patients, such as cyclosporine (CSA), methotrexate (MTX), azathioprine (AZA), mycophenolate mofetil (MMF), tacrolimus, cyclophosphamide, and biological agents (adalimumab, infliximab, rituximab, IFN- $\beta$, and IFN- $\alpha 2 a)^{14,40-42}$. Their use is supported by evidence generated in several randomized controlled trials ${ }^{14,43}$, attesting their effectivity and safety in NINA-U. It bears mentioning that, with the exception of CSA in few European countries, infliximab in Japan, and adalimumab in US and the EU, no other IMDs have regulatory approval for their prescription in NINA-U. 
Furthermore, several observational retrospective studies have outlined, in daily clinical practice, the effectiveness of several IMDs used in monotherapy ${ }^{44-47}$. These studies have shown different rates of clinical outcomes, such as achievement of inactivity, corticosteroid sparing, and remission, for the different drugs. However, these and others studies have underlined that in a percentage of patients, the use of IMDs in monotherapy was unable to achieve a sustained control of the inflammatory process: Joshi et al ${ }^{48}$ reported that a large proportion of uveitis patients initially prescribed with IMDs in monotherapy underwent several changes in their immunosuppressive treatment overtime (40$75 \%$ of patients had to discontinue their first IMD due to ineffectiveness or serious adverse events). In addition, $55 \%$ required a change in their initial treatment. Menezo et al. ${ }^{49}$ showed that $32 \%$ of patients in IMD monotherapy required the addition of a second drug. Goldberg et al. ${ }^{50}$ reported in patients with multifocal choroidopathies initially treated with MMF that at 24 months follow-up, $21 \%$ required the addition of a second IMD to control inflammation. Data from Abasolo et al. ${ }^{51}$ showed that among patients initially receiving IMDs in monotherapy, $46.2 \%$ required a modification in their initial treatment $(27.4 \%$ of patients added a second IMD due to inefficacy and $18.9 \%$ switched to a new IMD).

Despite the brief discussion in the latest international consensus document regarding NIU management $^{14}$, the combination of two or more IMDs could offer advantages over monotherapy, increasing effectiveness by addressing different therapeutic pathways and decreasing the incidence of adverse events, as the combinations could allow the prescription of a lower dose of the drugs combined ${ }^{52}$. In fact, the effectiveness and tolerability of combination therapy with IMDs for the treatment of NINA-U ${ }^{53-55}$, as well as other immune-mediated inflammatory diseases, such as rheumatoid arthritis ${ }^{56}$, have been evaluated in several observational retrospective case reports/series or open label studies including several conditions: Pascalis et al. ${ }^{57}$ analyzed intermediate and posterior uveitis patients resistant to IMD monotherapy and high corticosteroids dosage, who were treated with CSA and MTX in combination, observing a good response in most patients, both in control of the inflammation and visual acuity improvement. In Birdshot retinochoroidopathy, Cervantes-Castañeda et al. ${ }^{53}$ reported that the combination of CSA and MMF was able to induce inactivity in $63 \%$ of patients during the first 12 months of follow-up, all being able to discontinue the systemic corticosteroid treatment. In addition, the probability of no vitreous inflammation was $81 \%$ at 12 months. In serpiginous choroiditis, Hooper et al. ${ }^{55}$ reported five patients successfully treated with a combination of CSA, AZA, and systemic corticosteroids, with a rapid and maintained induction of inactivity. In addition, Akpek et al. ${ }^{54}$ reported six patients with the same condition, treated with a combination of CSA, AZA, and cyclophosphamide, who achieve control of inflammation. In VogtKoyanagi-Harada syndrome, five patients treated with systemic corticosteroids, AZA and CSA showed a rapid control of the inflammatory process, and decreased the frequency of recurrences during follow-up ${ }^{58}$. Arcinue et al. ${ }^{59}$ also reported the successful use of this combination and of CSA and MMF in these patients. In ocular Behçet's disease, combination of cyclophosphamide and AZA showed efficacy in 295 patients $^{60}$. The combination of CSA and infliximab was also proven successful ${ }^{61}$. Despite the evidence from observational studies, a direct comparison between combination and monotherapy has not been published yet, although some groups have adopted the use of combination therapy as the initial IMD treatment for particular conditions ${ }^{62}$, and a randomized clinical trial is being carried out to assess the efficacy and safety of this therapeutic approach ${ }^{63}$.

\section{Conclusions}

NINA-U are a heterogeneous group of mostly chronic or relapsing diseases that can lead to vision loss and produce high levels of disability and a reduction in the quality of life. To prevent these negative outcomes, careful pharmacological management is needed, with the objective of a complete and maintained suppression of the inflammatory process. Corticosteroids, administered using different routes based on the clinical manifestation and diagnosis, are the cornerstone of treatment, due to their almost immediate effect. Furthermore, IMDs in monotherapy or in combination can, and in several instances should, be combined with corticosteroids to ensure a maintained control of the inflammatory manifestations and prevent the arise of permanent ocular structural damage leading to irreversible vision loss.

\section{Funding}

None. 


\section{Conflicts of interest}

None.

\section{Ethical disclosures}

Protection of human and animal subjects. The authors declare that no experiments were performed on humans or animals for this study.

Confidentiality of data. The authors declare that no patient data appear in this article.

Right to privacy and informed consent. The authors declare that no patient data appear in this article.

\section{References}

1. Jabs DA, Nussenblatt RB, Rosenbaum JT. Standardization of uveitis nomenclature for reporting clinical data. Results of the first international workshop. Am J Ophthalmol. 2005;140:509-16.

2. Caspi RR. A look at autoimmunity and inflammation in the eye. $\mathrm{J}$ Clin Invest. 2010;120:3073-83.

3. Márquez A, Cordero-Coma M, Martín-Villa JM, Gorroño-Echebarría MB Blanco R, Valle DD, et al. New insights into the genetic component of non-infectious uveitis through an immunochip strategy. J Med Genet. 2017:54:38-46.

4. Lee RW, Nicholson LB, Sen HN, Chan CC, Wei L, Nussenblatt RB, et al Autoimmune and autoinflammatory mechanisms in uveitis. Semin Immunopathol. 2014;36:581-94.

5. Willermain F, Rosenbaum JT, Bodaghi B, Rosenzweig HL, Childers S, Behrend $\mathrm{T}$, et al. Interplay between innate and adaptive immunity in the development of non-infectious uveitis. Prog Retin Eye Res. 2012;31:182-94.

6. World Health Organization. The World Health Report, Global Data on Visual Impairments; 2010. Available from: http://www.who.int/blindness/ GLOBALDATAFINALforweb.pdf.

7. Suttorp-Schulten MS, Rothova A. The possible impact of uveitis in blindness: a literature survey. Br J Ophthalmol. 1996;80:844-8.

8. de Smet MD, Taylor SR, Bodaghi B, Miserocchi E, Murray PI, Pleyer U, et al. Understanding uveitis: the impact of research on visual outcomes. Prog Retin Eye Res. 2011;30:452-70

9. Rothova A, Suttorp-van Schulten MS, Frits Treffers W, Kijlstra A. Causes and frequency of blindness in patients with intraocular inflammatory disease. Br J Ophthalmol. 1996;80:332-6.

10. Arriola-Villalobos P, Abásolo L, García-Feijoo J, Fernández-Gutiérrez B, Méndez-Fernández R, Pato $\mathrm{E}$, et al. Vision-related quality of life in patients with non-infectious uveitis: a cross-sectional study. Ocul Immunol Inflamm. 2017;26:717-25.

11. Suhler EB, Lloyd MJ, Choi D, Rosenbaum JT, Austin DF. Incidence and prevalence of uveitis in veterans affairs medical centers of the Pacific Northwest. Am J Ophthalmol. 2008;146:890-6.e8.

12. Jalil A, Yin K, Coyle L, Harper R, Jones NP. Vision-related quality of life and employment status in patients with uveitis of working age: a prospective study. Ocul Immunol Inflamm. 2012;20:262-5.

13. Durrani OM, Tehrani NN, Marr JE, Moradi P, Stavrou P, Murray PI. Degree, duration, and causes of visual loss in uveitis. $\mathrm{Br} \mathrm{J}$ Ophthalmol. 2004;88:1159-62

14. Dick AD, Rosenbaum JT, Al-Dhibi HA, Belfort R Jr., Brézin AP, Chee SP, et al. Guidance on noncorticosteroid systemic immunomodulatory therapy in noninfectious uveitis: fundamentals of care for uveitis (FOCUS) initiative. Ophthalmology. 2018:125:757-73.

15. Gregory AC, Kempen JH, Daniel E, Kaçmaz RO, Foster CS, Jabs DA, et al. Risk factors for loss of visual acuity among patients with uveitis associated with juvenile idiopathic arthritis: the systemic immunosuppressive therapy for eye diseases study. Ophthalmology. 2013;120:186-92.

16. Kaçmaz RO, Kempen JH, Newcomb C, Gangaputra S, Daniel E, Levy-Clarke GA, et al. Ocular inflammation in Behçet disease: incidence of ocular complications and of loss of visual acuity. Am J Ophthalmol. 2008;146:828-36.

17. Nguyen QD, Callanan D, Dugel P, Godfrey DG, Goldstein DA, Wilensky JT. Treating chronic noninfectious posterior segment uveitis: the impact of cumulative damage. Proceedings of an expert panel roundtable discussion. Retina. 2006;2006:1-16.
18. Wakefield D, McCluskey P, Wildner G, Thurau S, Carr G, Chee SP, et al. Inflammatory eye disease: pre-treatment assessment of patients prior to commencing immunosuppressive and biologic therapy: recommendations from an expert committee. Autoimmun Rev. 2017;16:213-22.

19. Smith JR, Rosenbaum JT. Management of uveitis: a rheumatologic perspective. Arthritis Rheum. 2002;46:309-18.

20. Gaudio PA. A review of evidence guiding the use of corticosteroids in the treatment of intraocular inflammation. Ocul Immunol Inflamm. 2004;12:169-92.

21. Burkholder BM, Jabs DA. Uveitis for the non-ophthalmologist. BMJ. 2021;372:m4979.

22. Carnahan MC, Goldstein DA. Ocular complications of topical, peri-ocular, and systemic corticosteroids. Curr Opin Ophthalmol. 2000;11:478-83.

23. Riordan-Eva $P$, Lightman $S$. Orbital floor steroid injections in the treatment of uveitis. Eye. 1994;8:66-9.

24. Weijtens O, Schoemaker RC, Lentjes EG, Romijn FP, Cohen AF, van Meurs JC, et al. Dexamethasone concentration in the subretinal fluid after a subconjunctival injection, a peribulbar injection, or an oral dose. Ophthalmology. 2000;107:1932-8.

25. Bleriot A, Couret C, Le Meur G, Lebranchu P, Weber M. Safety and efficacy of subconjunctival triamcinolone injections in the management of uveitic macular edema: retrospective study of thirty-one cases. J Fr Ophtalmol. 2014;37:599-604.

26. Sen HN, Vitale S, Gangaputra SS, Nussenblatt RB, Liesegang TL, Levy-Clarke GA, et al. Periocular corticosteroid injections in uveitis: effects and complications. Ophthalmology. 2014;121:2275-86.

27. Sallam A, Taylor SR, Habot-Wilner Z, Elgohary M, Do HH, McCluskey P, et al. Repeat intravitreal triamcinolone acetonide injections in uveitic macular oedema. Acta Ophthalmol. 2012;90:e323-5.

28. Androudi S, Letko E, Meniconi M, Papadaki T, Ahmed M, Foster CS. Safety and efficacy of intravitreal triamcinolone acetonide for uveitic macular edema. Ocul Immunol Inflamm. 2005;13:205-12

29. Lowder C, Belfort R, Lightman S, Foster CS, Robinson MR, Schiffman RM, et al. Dexamethasone intravitreal implant for noninfectious intermediate or posterior uveitis. Arch Ophthalmol. 2011;129:545-53.

30. Thorne JE, Sugar EA, Holbrook JT, Burke AE, Altaweel MM, Vitale AT, et al. Periocular triamcinolone vs. Intravitreal triamcinolone vs. Intravitreal dexamethasone implant for the treatment of uveitic macular edema: the PeriOcular vs. INTravitreal corticosteroids for uveitic macular edema (POINT) trial. Ophthalmology. 2019;126:283-95.

31. Callanan DG, Jaffe GJ, Martin DF, Pearson PA, Comstock TL. Treatment of posterior uveitis with a fluocinolone acetonide implant: three-year clinical trial results. Arch Ophthalmol. 2008;126:1191-201.

32. Multicenter Uveitis Steroid Treatment (MUST) Trial Follow-up Study Research Group. Quality of life and risks associated with systemic anti-inflammatory therapy versus fluocinolone acetonide intraocular implant for intermediate uveitis, posterior uveitis, or panuveitis: fifty-four-month results of the multicenter uveitis steroid treatmen. Ophthalmology. 2015;122:1976-86.

33. Jaffe GJ, Pavesio CE, Study Investigators. Effect of a fluocinolone acetonide insert on recurrence rates in noninfectious intermediate, posterior, or panuveitis: three-year results. Ophthalmology. 2020;127:1395-404.

34. Jabs DA. Treatment of ocular inflammation. Ocul Immunol Inflamm. 2004;12:163-8.

35. Da Silva JA, Jacobs JW, Kirwan JR, Boers M, Saag KG, Inês LB, et al. Safety of low dose glucocorticoid treatment in rheumatoid arthritis: published evidence and prospective trial data. Ann Rheum Dis. 2006;65:285-93.

36. Roujeau JC. Pulse glucocorticoid therapy. The "Big Shot" revisited. Arch Dermatol. 1996;132:1499-502.

37. Charkoudian LD, Ying G, Pujari SS, Gangaputra S, Thorne JE, Foster CS, et al. High-dose intravenous corticosteroids for ocular inflammatory diseases. Ocul Immunol Inflamm. 2012;20:91-9.

38. Nakayama $M$, Keino $H$, Watanabe $T$, Okada AA. Clinical features and visual outcomes of 111 patients with new-onset acute Vogt-Koyanagi-Harada disease treated with pulse intravenous corticosteroids. $\mathrm{Br} \mathrm{J}$ Ophthalmol. 2019;103:274-8.

39. Read RW, Yu F, Accorinti M, Bodaghi B, Chee SP, Fardeau C, et al. Evaluation of the effect on outcomes of the route of administration of corticosteroids in acute Vogt-Koyanagi-Harada disease. Am J Ophthalmol. 2006:142:119-24.

40. Jabs D a, Rosenbaum JT, Foster CS, Holland GN, Jaffe GJ, Louie JS, et al. Guidelines for the use of immunosuppressive drugs in patients with ocular inflammatory disorders: recommendations of an expert panel. Am J Ophthalmol. 2000;130:492-513.

41. Becker MD, Smith JR, Max R, Fiehn C. Management of sight-threatening uveitis: new therapeutic options. Drugs. 2005;65:497-519.

42. Jabs DA. Immunosuppression for the Uveitides. Ophthalmology. 2018;125:193-202

43. Gómez-Gómez A, Loza E, Rosario MP, Espinosa G, de Morales JM, Herrera $\mathrm{JM}$, et al. Efficacy and safety of immunomodulatory drugs in patients with non-infectious intermediate and posterior uveitis, panuveitis and macular edema: a systematic literature review. Semin Arthritis Rheum. 2020;50:1299-306. 
44. Pasadhika S, Kempen JH, Newcomb CW, Liesegang TL, Pujari SS Rosenbaum JT, et al. Azathioprine for ocular inflammatory diseases. Am J Ophthalmol. 2009;148:500-9.e2.

45. Gangaputra S, Newcomb CW, Liesegang TL, Kaçmaz RO, Jabs DA Levy-Clarke GA, et al. Methotrexate for ocular inflammatory diseases. Ophthalmology. 2009;116:2188-98.e1.

46. Daniel E, Thorne JE, Newcomb CW, Pujari SS, Kaçmaz RO, Levy-Clarke GA, et al. Mycophenolate mofetil for ocular inflammation. Am J Ophthalmol. 2010;149:423-32.e1-2.

47. Kaçmaz RO, Kempen JH, Newcomb C, Daniel E, Gangaputra S, Nussenblatt RB, et al. Cyclosporine for ocular inflammatory diseases. Ophthalmology. 2010;117:576-84.

48. Joshi L, Talat L, Yaganti S, Sandhu S, Taylor SR, Wakefield D, et al. Outcomes of changing immunosuppressive therapy after treatment failure in patients with noninfectious uveitis. Ophthalmology. 2014; 121:1119-24

49. Menezo V, Lau C, Comer M, Lightman S. Clinical outcome of chronic immunosuppression in patients with non-infectious uveitis. Clin Exp Ophthalmol. 2005;33:16-21.

50. Goldberg NR, Lyu T, Moshier E, Godbold J, Jabs DA. Success with single-agent immunosuppression for multifocal choroidopathies. Am J Ophthalmol. 2014;158:1310-7.

51. Abásolo L, Rosales Z, Díaz-Valle D, Gómez-Gómez A, Peña-Blanco RC Prieto-García A, et al. Immunosuppressive drug discontinuation in noninfectious uveitis from real-life clinical practice: a survival analysis. Am J Ophthalmol. 2016;169:1-8.

52. Vitale AT, Rodriguez A, Foster CS. Low-dose cyclosporine therapy in the treatment of birdshot retinochoroidopathy. Ophthalmology. 1994; 101:822-31

53. Cervantes-Castañeda RA, Gonzalez-Gonzalez LA, Cordero-Coma M, Yilmaz T, Foster CS. Combined therapy of cyclosporine A and mycophenolate mofetil for the treatment of birdshot retinochoroidopathy: a 12-month follow-up. Br J Ophthalmol. 2013;97:637-43.
54. Akpek EK, Baltatzis S, Yang J, Foster CS. Long-term immunosuppressive treatment of serpiginous choroiditis. Ocul Immunol Inflamm. 2001;9:153-67.

55. Hooper PL, Kaplan HJ. Triple agent immunosuppression in serpiginous choroiditis. Ophthalmology. 1991;98:944-51.

56. Hazlewood GS, Barnabe C, Tomlinson G, Marshall D, Devoe D, Bombardier $\mathrm{C}$. Methotrexate monotherapy and methotrexate combination therapy with traditional and biologic disease modifying antirheumatic drugs for rheumatoid arthritis: abridged Cochrane systematic review and network meta-analysis. BMJ. 2016;353:i1777.

57. Pascalis L, Pia G, Aresu G, Frongia T, Barca L. Combined cyclosporin A-steroid-MTX treatment in endogenous non-infectious uveitis. J Autoimmun. 1993;6:467-80

58. Agarwal M, Ganesh SK, Biswas J. Triple agent immunosuppressive therapy in Vogt-Koyanagi-Harada syndrome. Ocul Immunol Inflamm. 2006;14:333-9.

59. Arcinue CA, Radwan A, Lebanan MO, Foster CS. Comparison of two different combination immunosuppressive therapies in the treatment of Vogt-Koyonagi-Harada syndrome. Ocul Immunol Inflamm. 2013:21:47-52.

60. Davatchi F, Sadeghi Abdollahi B, Shams H, Shahram F, Nadji A, Chams-Davatchi $C$, et al. Combination of pulse cyclophosphamide and azathioprine in ocular manifestations of Behcet's disease: Iongitudinal study of up to 10 years. Int J Rheum Dis. 2014;17:444-52.

61. Katsuyama A, Kusuhara S, Nishisho R, Matsumiya W, Azumi A, Nakamura M. Long-term efficacy and safety of infliximab and cyclosporine combination therapy for refractory uveoretinitis in Behçet's disease. Clin Ophthalmol. 2019;13:521-7.

62. Kiss S, Ahmed M, Letko E, Foster CS. Long-term follow-up of patients with birdshot retinochoroidopathy treated with corticosteroid-sparing systemic immunomodulatory therapy. Ophthalmology. 2005;112:1066-71.

63. Efficacy, Safety and Costs of Methotrexate, Adalimumab, or Their Combination in Non-infectious Non-anterior Uveitis (Co-THEIA); 2021. Available from: https://www.clinicaltrials.gov/ct2/show/NCT04798755. [Last accessed on 2021 Aug 19]. 